\title{
A Lower Bound on the per Soliton Capacity of the Nonlinear Optical Fibre Channel
}

\author{
Nikita A. Shevchenko*, Jaroslaw E. Prilepsky ${ }^{\dagger}$, Stanislav A. Derevyanko ${ }^{\S}$, \\ Alex Alvarado*, Polina Bayvel* and Sergei K. Turitsyn ${ }^{\dagger}$ \\ * Optical Networks Group, University College London, Torrington Place, London WC1E 7JE, United Kingdom \\ ${ }^{\dagger}$ Aston Institute of Photonic Technologies, Aston University, Birmingham B4 7ET, United Kingdom \\ $\S$ Department of Physics of Complex Systems, Weizmann Institute of Science, Rehovot 76100, Israel
}

\begin{abstract}
A closed-form expression for a lower bound on the per soliton capacity of the nonlinear optical fibre channel in the presence of (optical) amplifier spontaneous emission (ASE) noise is derived. This bound is based on a non-Gaussian conditional probability density function for the soliton amplitude jitter induced by the ASE noise and is proven to grow logarithmically as the signal-to-noise ratio increases.
\end{abstract}

\section{INTRODUCTION}

It is widely accepted that in order to meet the ever-growing demand for data rates in fibre-optic telecommunication systems, the spectral efficiency of the optical fibre transmission system needs to be increased [1]. The key physical effects distinguishing a fibre optical system from a free space transmission are: dispersion, nonlinearity and optical noise [2][5]. The implementation of the "fifth generation" of optical transceivers and networks operating with coherent detection, advanced multilevel modulation formats, and digital signal processing techniques, has led to the possibility of channel rates exceeding $100 \mathrm{Gbit} / \mathrm{s}[6]$. The key to this breakthrough is the mitigation of linear transmission impairments, such as chromatic and polarization mode dispersion.

The performance of current coherent systems is limited by noise and nonlinearity. In contrast to linear channels, however, spectral efficiencies for the optical fibre channel usually exhibit a peak and decay at high input powers; this is often referred to as the "nonlinear Shannon limit" [7], [8]. This behaviour is caused by the Kerr nonlinearity and is believed to ultimately lead to a "capacity crunch" [1], i.e., to the inability of the optical network infrastructure to cope with the increasing capacity demand.

The capacity analysis of the nonlinear channel relies on well-established methods of information theory [9], [10]. However, most of the analytical results obtained to date concern linear channel models, and hence, are not directly applicable to nonlinear optical channels. Despite numerous efforts to define the influence of Kerr nonlinearity on the channel capacity [7], [8], [11]-[16], the capacity of the nonlinear optical channel still remains as an open research problem. Most of the capacity bounds presented in the literature typically display a peaky

Research supported by the Engineering and Physical Sciences Research Council (EPSRC) project UNLOC (EP/J017582/1), United Kingdom. behaviour, where the maximum is reached at a finite threshold power. To the best of our knowledge, the first nondecaying (lower) bound on the capacity of the nonlinear optical fibre channel (with zero average dispersion) was presented in [17]. Other nondecaying bounds include, e.g., those given recently in [18] and [19], [20].

A multitude of different nonlinearity mitigation techniques have been proposed over recent years to suppress nonlinearityinduced distortions. This includes receiver-based digital signal processing [21], digital back-propagation [22], optical phase conjugation [23], twin-waves phase conjugation [24], etc. However, there are still many limitations and further challenges in applying these methods. A promising alternative for nonlinearity compensation is the nonlinear Fourier transform (NLFT) developed in the 70's [25], [26]. The applications of the NLFT in optical communication originates from the pioneering work of Hasegawa and Nyu [27], an approach that has been extended in a number of recent works [28][37]. Notably, an experimental demonstration of a NLFT-based transmission was recently presented by Bülow in [38].

The use of NLFT for nonlinearity compensation in optical fibre links is possible because the master model governing signal propagation in a single mode optical fibre (in the absence of noise and loss) is the nonlinear Schrödinger equation (NLSE) [3]-[5] that belongs to the class of integrable (i.e., completely solvable) evolutionary equations [25]. The solution method can be considered as the generalisation of the linear Fourier transform (FT) operation onto the nonlinear (integrable) system, hence the name NLFT. Similarly to the FT, the NLFT decomposes a waveform in the NLSE space-time domain into the nonlinear normal modes inside the nonlinear spectral domain [29], [35]. The key underlying feature of the NLFT transmission is that these nonlinear modes (nonlinear signal spectrum) propagate without cross-talk, effectively in a linear manner. Thus, the nonlinear spectrum can be used for encoding and efficient transmission of information over the nonlinear fibre.

The original work by Hasegawa and Nyu [27] introduced the concept of "eigenvalue communications", where the information was encoded using discrete eigenvalues associated with the solitonic degrees of freedom emerging from the NLFT 
signal decomposition [3] (see also [29]). In the absence of both loss and noise, the evolution of nonlinear modes is inherently free from any nonlinear impairments, including a nonlinear cross-talk. The loss in optical links is usually compensated by using lumped or distributed amplification; in our case we assume the ideal distributed Raman amplification scheme resulting in the lossless NLSE [2], [39], [40]. However, the signal will still be distorted by amplifier-induced spontaneous emission (ASE) as well as signal-noise beating.

In this paper, we study the channel capacity (in bits per soliton symbol 11 for a transmission system based on optical solitons (sufficiently separated in time domain) launched into a noisy NLSE channel. The information is assumed to be encoded in the soliton's amplitude only, which can be extracted from the imaginary part of the discrete eigenvalue emerging from the NLFT signal decomposition. We consider a discretetime continuous-input continuous-output channel model, based on the asymptotically exact non-Gaussian marginal statistics of the soliton amplitude in the presence of weak ASE noise presented in [41]-[43]. We emphasise that the capacity estimations for such fundamentally nonlinear channels are quite few and far between. Notable exceptions are the works by Yousefi and Kschischang [31] and Meron et al. [33]. While in [31] the channel statistics were assumed a priori to be Gaussian [31, eq. (27)], in [33] a tight lower bound on the channel capacity as a function of the signal to noise ratio (SNR) was not provided.

The discrete-time channel model governing transmission systems based on optical solitons is a noncentral chi-squared distribution with four degrees of freedom [42], [43]. Based on this model we obtain an asymptotically growing lower bound for the channel capacity vs. SNR. This bound is similar to the one in [17], where a noisy nonlinear optical fibre channel with zero fibre dispersion was considered. The results in this paper show that the reachable capacity limits for existing optical fibre channels could have been previously underestimated.

\section{The Master Equation AND the Noncentral CHI-SQuared Channel Model}

\section{A. Waveform channel}

We consider propagation of a slowly varying envelope signal formed by a sequence of solitons transmitted every $T_{s}$ [s] over a nonlinear optical fibre. Our model combines the effects of chromatic dispersion (we consider the case of anomalous dispersion), instantaneous Kerr nonlinearity, and ASE noise due to optical Raman amplification. The fibre loss is assumed to be continuously compensated along the fibre by means of ideal Raman amplification and hence is set to zero [2], [39], [40].

The noise-perturbed NLSE in dimensionless units is given by [2], [3], [42]

$$
i \frac{\partial q(z, t)}{\partial z}+\frac{1}{2} \frac{\partial^{2} q(z, t)}{\partial t^{2}}+|q(z, t)|^{2} q(z, t)=n(z, t),
$$

\footnotetext{
${ }^{1}$ The practically more relevant problem of channel capacity in $[\mathrm{bit} / \mathrm{s} / \mathrm{Hz}]$ is left for future investigation.
}

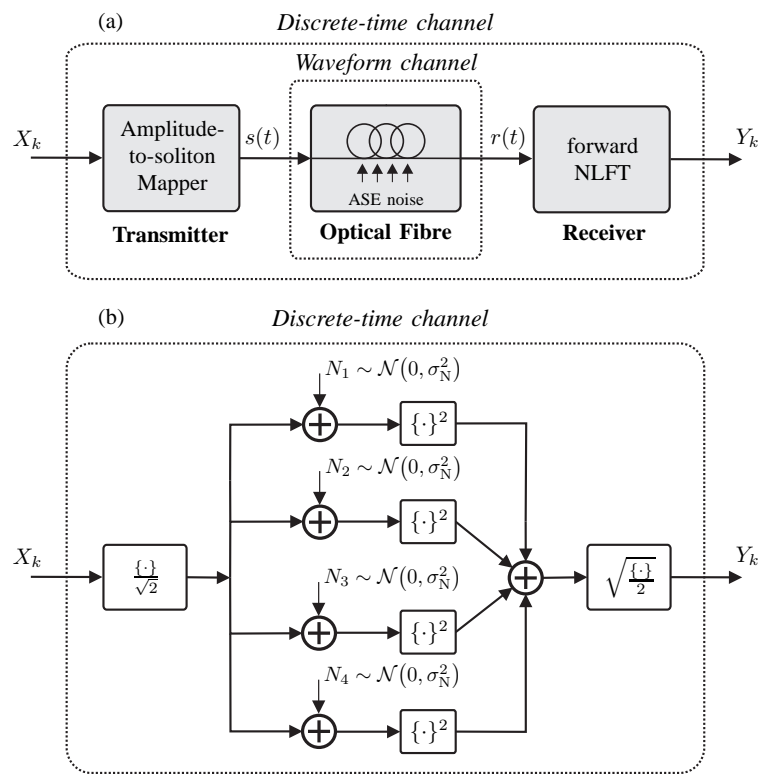

Fig. 1. System model: (a) Transmitter, continuous-channel model governed by 1 , and receiver. (b) Equivalent discrete-time channel model.

where $t$ is the time normalised by $T_{s}, z$ is the distance along the fibre normalised by $L_{s} \triangleq \frac{T_{s}^{2}}{\left|\beta_{2}\right|}$ (not to be confused with the dispersion length), and $\beta_{2}<0$ is the group velocity dispersion coefficient. We also define $s(t)=q(0, t)$ and $r(t)=q(L, t)$ as the input and output waveforms of the physical channel after transmission distance $L$, respectively, normalised by the nonlinear power scale $\left(\gamma L_{s}\right)^{-1}$, where $\gamma$ is the nonlinearity coefficient. The relationship between $s(t)$ and $r(t)$ is schematically shown in the inner part of Fig. 11 a).

The noise term $n(t, z)$ on the right-hand side of (1) is assumed to be a zero-mean $(\mathbb{E}[n(z, t)]=0)$ circularlysymmetric additive white Gaussian noise (AWGN) process with autocorrelation function [2, eq. (53)]

$$
\mathbb{E}\left[n(z, t) n^{*}\left(z^{\prime}, t^{\prime}\right)\right]=2 D \delta\left(z-z^{\prime}\right) \delta\left(t-t^{\prime}\right),
$$

where * denotes complex conjugation, $\mathbb{E}[\cdot]$ is the mathematical expectation operator, and $\delta(\cdot)$ is the Dirac's delta function. Here the noise intensity $D$ is written in dimensionless units [4, eq. (5.29)] as $D=\gamma L_{s}^{2} \sigma_{0}^{2} / 2 T_{s}$. For ideal distributed Raman amplification, the power spectral density of the ASE noise $\sigma_{0}^{2}$ is defined as [2, eq. (56)] $\sigma_{0}^{2}=\alpha K_{T} \cdot h \nu_{\text {opt }}$, where $\alpha$ is the fibre attenuation coefficient, $h \nu_{\mathrm{opt}}$ is the average photon energy, and $K_{T} \approx 1$ is the coefficient that characterizes the Raman pump providing the gain [2].

It is known that the noiseless NLSE (i.e., (1) with $n(z, t)=$ 0 ) possesses a special class of solutions, the so-called fundamental bright solitons [3]-[5]. At $z=0$ we write it as [5, eq. (1.40)] (in normalised units)

$$
q(0, t)=A_{0} \operatorname{sech}\left(A_{0} t\right),
$$

where $A_{0}$ denotes the normalised soliton amplitude and we assume that the initial soliton frequency, phase and centreof-mass position are set to zero. The unperturbed soliton solution (3) at a distance $z=L$ is given by $q(L, t)=$ $A_{0} \operatorname{sech}\left(A_{0} t\right) \exp \left(i A_{0}^{2} L / 2\right)$. 


\section{B. Discrete-time channel}

The (normalised) continuous-time input signal $s(t)$ is defined as

$$
s(t)=\sum_{k=1}^{\infty} s_{k}(t)
$$

where

$$
s_{k}(t)=A_{0 k} \operatorname{sech}\left[A_{0 k}(t-k)\right],
$$

and $k$ is the discrete-time index. At each discrete time $k$, the transmitter maps an amplitude $A_{0 k}$ to $s_{k}(t)$ via (5). For simplicity of the following analysis, however, we consider the square root of the amplitudes, i.e., $X_{k}=\sqrt{A_{0 k}}$.

The dimensionless energy of the $k$ th soliton waveform is defined as

$$
E\left(A_{0 k}\right) \triangleq \int_{-(k-1 / 2)}^{(k+1 / 2)}\left|s_{k}(t)\right|^{2} \mathrm{~d} t .
$$

We consider the regime where the inter-soliton separation $T_{s}$ is much larger than the typical soliton width (low duty cycle), so the integral in (6) can be taken over $(-\infty, \infty)$. This yields the well-known linear energy-amplitude scaling of the soliton pulse $E\left(A_{0 k}\right)=2 A_{0 k}$. The minimum inter-soliton separation is then determined by the peak power $A_{0 k}^{2}$ of each individual soliton, which is in turn inversely proportional to the square of its width $T_{0 k}=\frac{1}{A_{0 k}}$.

The receiver in Fig. 1(b) processes the received waveform $r(t)$ during a window of length one via the forward NLFT and returns the amplitude of the received soliton. We assume ideal NLFT-detection, i.e., the sampling rate is high enough to ignore NLFT finite accuracy issues arising from a particular algorithmic realisation [30] compared to noise-induced distortions. The inter-soliton separation is also assumed to be large enough so that there is no interaction between adjacent solitons, i.e., $\exp \left(-A_{0 k}\right) \ll 1$, or equivalently, $1 \gg T_{0 k}$. Another source of corruption for the soliton-based transmission system emanates from the Gordon-Haus (GH) timing jitter [5], [33], which defines the standard deviation $\Delta T^{\mathrm{GH}}$ of the soliton position as a function of the propagation distance and soliton amplitude. To avoid interaction between adjacent solitons, the GH timing jitter should also be taken into account [5]. For a given propagation distance $L$, the inter-soliton separation must fulfill $1>T_{0 k}+\Delta T^{\mathrm{GH}}$. This condition guarantees that solitons behave as isolated pulses, and thus, there is no intersymbol interference. We assume that this condition is satisfied throughout this paper, and thus, from now on we drop the time index $k$.

The exact conditional PDF for a single received amplitude $A$ given a transmitted amplitude $A_{0}$ is written as [42, eq. (24)] (see also [43])

$$
p_{A \mid A_{0}}\left(a \mid a_{0}\right)=\frac{1}{\sigma_{\mathrm{N}}^{2}} \sqrt{\frac{a}{a_{0}}} \exp \left(-\frac{a_{0}+a}{\sigma_{\mathrm{N}}^{2}}\right) I_{1}\left(\frac{2 \sqrt{a_{0} a}}{\sigma_{\mathrm{N}}^{2}}\right),
$$

where $\sigma_{\mathrm{N}}^{2}=L L_{s}^{-1} D / 2$ is the normalised variance of the accumulated ASE noise and $I_{1}(x)$ is the modified Bessel function of the first kind. Expression (7) is in fact the same
PDF obtained assuming an energy-detection receiver (i.e., a receiver based on (6)), as shown in [4, eq. (5.501)].

Equation (7) is nothing else but a special case of a noncentral chi-squared distribution with four degrees of freedom providing non-Gaussian statistics for soliton amplitudes. For future use, it is convenient to designate the output of the discrete-time channel model $Y$ as the square root of the output soliton amplitudes $A$. By making a change of variables, the PDF (7) can be rewritten as

$$
p_{Y \mid X}(y \mid x)=\frac{2}{\sigma_{\mathrm{N}}^{2}} \frac{y^{2}}{x} \exp \left(-\frac{x^{2}+y^{2}}{\sigma_{\mathrm{N}}^{2}}\right) I_{1}\left(\frac{2 x y}{\sigma_{\mathrm{N}}^{2}}\right) .
$$

The conditional PDF in (8) describes a channel with the input-output relation

$$
Y^{2}=\frac{1}{2} \sum_{i=1}^{4}\left(\frac{X}{\sqrt{2}}+N_{i}\right)^{2},
$$

where $N_{i}, i=1,2,3,4$ are four independent and identically distributed zero-mean Gaussian random variables with variance $\sigma_{i}^{2}=\sigma_{\mathrm{N}}^{2}$. The input-output relationship in (9) is schematically shown in Fig. 1(b).

\section{MAIN RESUltS}

Since the soliton pulses are assumed to be well separated and the intersymbol interference due to pulse interaction can be neglected, the model (8) describes a scalar memoryless channel. The channel capacity is then defined as [9], [10]

$$
C \triangleq \max _{p_{X}} I_{X Y},
$$

where $I_{X Y}$ is the mutual information (MI) and the optimization is performed over all possible input distributions $p_{X}$ with fixed average symbol energy $\mathbb{E}\left[E\left(A_{0}\right)\right]$. The MI $I_{X Y}$ can be decomposed as [9], [10]

$$
I_{X Y}=h_{Y}-h_{Y \mid X},
$$

where $h_{Y}$ and $h_{Y \mid X}$ are the output and conditional differential entropies, respectively.

The SNR is defined as [2, eq. (29)]

$$
\mathrm{SNR} \triangleq \frac{\mathbb{E}\left[E\left(A_{0}\right)\right]}{\sigma_{\mathrm{N}}^{2} T_{s}}=\frac{2 \kappa \sigma_{\mathrm{S}}^{2}}{\sigma_{\mathrm{N}}^{2}},
$$

where $\sigma_{\mathrm{S}}^{2}$ is the average amplitude $\sigma_{\mathrm{S}}^{2}=\mathbb{E}\left[A_{0}\right]=\mathbb{E}\left[X^{2}\right]$ and $\kappa$ is the ratio between the available bandwidth and the symbol rate $1 / T_{s}$. Thus, for a fixed bandwidth and symbol rate, the SNR is proportional to the parameter $\rho \triangleq \sigma_{\mathrm{S}}^{2} / \sigma_{\mathrm{N}}^{2}$. We shall henceforth consider the capacity and MI as a function of $\rho$.

The exact solution for the power constrained optimization problem (10) with the channel model (8) is unknown. To obtain a lower bound on the capacity, we shall assume the input symbols $X$ are drawn from a trial input distribution. In this work we use the Rayleigh PDF

$$
p_{X}(x)=\frac{2 x}{\sigma_{\mathrm{S}}^{2}} \exp \left(-\frac{x^{2}}{\sigma_{\mathrm{S}}^{2}}\right),
$$

which leads to exponentially-distributed soliton amplitudes $A_{0}$ with mean $\sigma_{\mathrm{S}}^{2}$. 
The next two Lemmas provide exact closed-form expressions for the output differential entropy $h_{Y}$ of symbols $Y$ with input symbols $X$ distributed according to (13) and for the conditional differential entropy $h_{Y \mid X}$.

Lemma 1: For the channel in (9) and the input distribution (13)

$$
\begin{aligned}
h_{Y}= & \log \sqrt{\sigma_{\mathrm{S}}^{2}}-\log \sqrt{1+\rho^{-1}}-\rho^{-1} \log \sqrt{1+\rho} \\
& +\rho+\psi\left(\rho^{-1}\right)-\frac{3}{2} \psi(1)-\log 2+1,
\end{aligned}
$$

where $\psi(x) \triangleq \frac{\mathrm{d}}{\mathrm{d} x} \ln \Gamma(x)$ is the digamma function, and $\Gamma(x)$ is the gamma function.

Lemma 2: For the channel in (9) and the input distribution (13)

$$
\begin{aligned}
h_{Y \mid X}= & \log \sqrt{\sigma_{\mathrm{S}}^{2}}+2(1+\rho)-\left(1+\rho^{-1}\right) \log (1+\rho) \\
& -\rho^{-1} \sqrt{1+\rho^{-1}} F(\rho)-\frac{\psi(1)}{2}-\log 2,
\end{aligned}
$$

where

$$
F(\rho) \triangleq \int_{0}^{\infty} \xi K_{1}\left(\sqrt{1+\rho^{-1}} \xi\right) I_{1}(\xi) \log \left[I_{1}(\xi)\right] \mathrm{d} \xi,
$$

and $K_{1}(x)$ is the modified Bessel function of the second kind of order one.

Sketch of the proof: To prove both lemmas, the output distribution $p_{Y}(y) \triangleq \int_{0}^{\infty} p_{\mathrm{Y} \mid \mathrm{X}}(y \mid x) p_{X}(x) \mathrm{d} x$ is calculated using (8) and (13). The derived output PDF $p_{Y}(y)$ is then used in the definitions of differential entropies. The results of both Lemmas are then obtained by evaluating the corresponding integrals. The calculation follows closely that from the earlier work [17], where calculations were performed for a chisquared distribution with two degrees of freedom (cf. (16) and [17, eq. (24)]).

We note that the proof of Lemma 2 includes finding a closed-form expression for the differential entropy of a chisquared distribution with four degrees of freedom. To the best of our knowledge, this has never been previously reported in the literature 2 The results from Lemmas 1 and 2 can be combined to produce the following theorem. (13)

Theorem 1: For the channel (9) and the input distribution

$$
\begin{aligned}
I_{X Y}= & \log \left(\rho \sqrt{1+\rho^{-1}}\right)+\rho^{-1} \log (\sqrt{1+\rho})-\rho \\
& +\rho^{-1} \sqrt{1+\rho^{-1}} F(\rho)+\psi\left(\rho^{-1}\right)-\psi(1)-1 .
\end{aligned}
$$

Proof: From Lemmas 1 and 2 and (11).

The results of Lemma 1, Lemma 2, and Theorem 1 are illustrated in Fig. 2 Analytical curves for the functions $h_{Y}, h_{Y \mid X}$, and $I_{X Y}$ are compared with results obtained via numerical integration.

\footnotetext{
${ }^{2}$ However, a closed-form expression for the expected-log of a noncentral chi-squared distribution with even number of degrees of freedom was given in [44. Lemma 10.1].
}

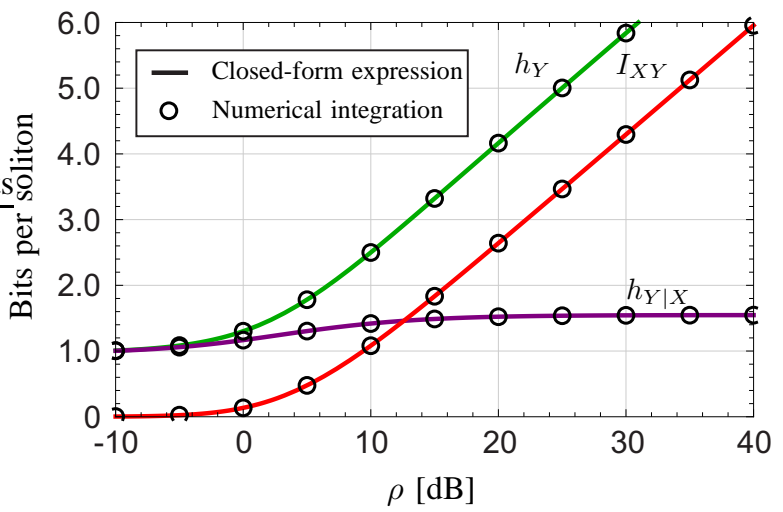

Fig. 2. The differential entropy of output distribution $h_{Y}$ in [14, the differential conditional entropy $h_{Y \mid X}$ in (15), and the MI $I_{X Y}$ in (17). Results obtained via numerical integration are also shown (circles).

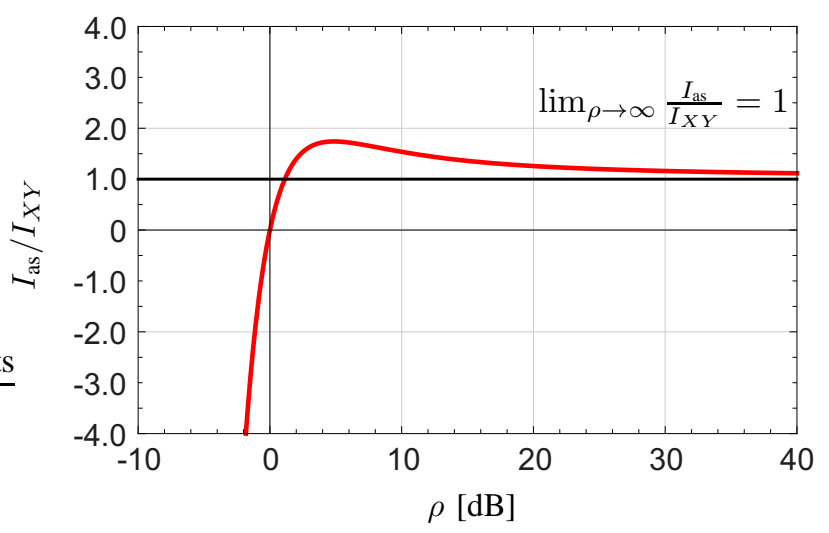

Fig. 3. The ratio between the MI $I_{X Y}$ in 17 and the function $I_{\text {as }}$ in 19 .

The next theorem shows that the capacity lower bound is asymptotically equivalent to half the logarithm of the SNR, which is the main result of our work.

Theorem 2: The MI $I_{X Y}$ in (17) satisfies

$$
\lim _{\rho \rightarrow \infty} \frac{I_{\mathrm{as}}}{I_{X Y}}=1,
$$

where

$$
I_{\mathrm{as}} \triangleq \frac{1}{2} \log \rho
$$

Proof: The proof follows from an asymptotic expansion of $I_{X Y}$ in (17) together with the asymptotic expansion of (16) provided in [17].

Fig. 3 shows the numerical evaluation of the ratio $I_{\text {as }} / I_{X Y}$ and confirms that the MI behaves asymptotically as $(1 / 2) \log \rho$, or equivalently, as $(1 / 2) \log$ SNR. According to Fig. 3, the asymptotic function (19) approaches the MI from above. Interestingly, the expression (19) has appeared in asymptotic analyses of optical systems (see e.g., [17, eq. (25)], [31, Sec. V-A], [45, eq. (6)]). Since the channel capacity is lower-bounded by $I_{X Y}$, this result implies that the capacity grows at least as fast as $(1 / 2) \log \mathrm{SNR}$, when $\mathrm{SNR} \rightarrow \infty$.

\section{CONClusions}

By using a rigorous channel model based on the exact conditional PDF for the soliton amplitudes in (7), an exact closed-form expression for a lower bound on the capacity of 
the nonlinear optical fibre channel with no inline dispersion compensation was derived. It has been analytically demonstrated that the lower bound on the capacity for the channel based on the individual amplitudes of well separated solitons displays an unbounded growth similarly to the linear Gaussian channel.

\section{REFERENCES}

[1] D. J. Richardson, "Filling the Light Pipe," Science, vol. 330, pp. $327-$ 328, Oct. 2010

[2] R. Essiambre, G. Kramer, P. J. Winzer, G. J. Foschini, and B. Goebel, "Capacity Limits of Optical Fiber Networks," J. Lightwave Technol., vol. 28, no. 4, pp. 662-701, Feb. 2010.

[3] A. Hasegawa and Y. Kodama, Solitons in Optical Communication, Clarendon Press, Oxford, 1995.

[4] E. Iannone, F. Matera, A. Mecozzi, and M. Settembre, Nonlinear Optical Communication Networks, Wiley, New York, 1998.

[5] L. F. Mollenauer, and J. P. Gordon, Solitons in Optical Fibers: Fundamentals and Applications, New York: Academic, 2006.

[6] M. Cvijetic and I. B. Djordjevic, Advanced Optical Communication Systems and Networks, Artech House, Norwood, MA, 2013.

[7] A. D. Ellis, J. Zhao, and D. Cotter, "Approaching the non-linear Shannon limit," J. Lightwave Technol., vol. 28, no. 4, pp. 423-433, Feb. 2010.

[8] A. Mecozzi and R. J. Essiambre, "Nonlinear Shannon Limit in Pseudolinear Coherent Systems," J. Lightwave Technol., vol. 30, no. 12, pp. 2011-2024, Jun. 2012.

[9] C. E. Shannon, "A Mathematical Theory of Communication," Bell Syst. Tech. J., vol. 27, no. 3/4, pp. 379-423/623-656, Jul./Oct. 1948.

[10] T. M. Cover and J. A. Thomas, Elements of Information Theory, 2nd ed., Hoboken, NJ: Wiley, 2006.

[11] A. Splett, C. Kurtzke and K. Petermann, "Ultimate Transmission Capacity of Amplified Optical Fiber Communication Systems Taking into Account Fiber Nonlinearities," in Proc. Eur. Conf. Opt. Commun., Montreux, Switzerland, Sept. 1993.

[12] P. Mitra and J. Stark, "Nonlinear Limits to the Information Capacity of Optical Fibre Communications," Nature, vol. 411, pp. 1027-1030, Jun. 2001.

[13] J. B. Stark, P. Mitra and A. Sengupta, "Information capacity of nonlinear wavelength division multiplexing fiber optic transmission line," Opt. Fiber Technol., vol. 7, pp. 275-288, Oct. 2001.

[14] L. G. L. Wegener et al., "The Effect of Propagation Nonlinearities on the Information Capacity of WDM Optical Fiber Systems: Cross-phase Modulation and Four-wave Mixing," Physica D: Nonlinear Phenomena, vol. 189, no. 1-2, pp. 81-99, Feb. 2004.

[15] M. Secondini, E. Forestieri, and G. Prati, "Achievable Information Rate in Nonlinear WDM Fiber-optic Systems with Arbitrary Modulation Formats and Dispersion Maps," J. Lightwave Technol., vol. 31, no. 23, pp. 3839-3852, Dec. 2013.

[16] R. Dar, M. Shtaif, M. Feder, "New bounds on the Capacity of the Nonlinear Fiber-optic Channel," Opt. Lett., vol. 39, no. 2, pp. 398-401, Jan. 2014.

[17] K. S. Turitsyn, S. A. Derevyanko, I. V. Yurkevich, and S. K. Turitsyn, "Information Capacity of Optical Fiber Channels with Zero Average Dispersion”, Phys. Rev. Lett., vol. 91, no. 30, art. no. 203901, Nov. 2003.

[18] E. Agrell, A. Alvarado, G. Durisi, and M. Karlsson, "Capacity of a Nonlinear Optical Channel With Finite Memory," J. Lightwave Technol., vol. 32, no. 16, pp. 2862-2876, Jun. 2014.

[19] G. Kramer, M. I. Yousefi, F. R. Kschischang, "Upper Bound on the Capacity of a Cascade of Nonlinear and Noisy Channels", in Proc. IEEE International Workshop on Information Theory, Jerusalem, Israel, AprMay 2015, available at http://arxiv.org/abs/1503.07652

[20] M. I. Yousefi, G. Kramer, F. R. Kschischang, "Upper Bound on the Capacity of the Nonlinear Schrödinger Channel," in Proc. Canadian Workshop on Information Theory, St. John's, Canada, available at http://arxiv.org/abs/1502.06455

[21] D. S. Millar, et al., "Mitigation of Fiber Nonlinearity Using a Digital Coherent Receiver," IEEE J. Sel. Top. Quantum Electron., vol. 16, pp. 1217-1226, Sept.-Oct. 2010.

[22] E. Ip and J. Kahn, "Compensation of Dispersion and Nonlinear Impairments Using Digital Backpropagation," J. Lightwave Technol., vol. 26, pp. 3416-3425, Oct. 2008.
[23] L. B. Du, M. M. Morshed, and A. J. Lowery, "Fiber Nonlinearity Compensation for OFDM Super-channels Using Optical Phase Conjugation," Opt. Express, vol. 20, pp. 19921-19927, Aug. 2012.

[24] X. Liu, A. R. Chraplyvy, P. J. Winzer, R. W. Tkach, and S. Chandrasekhar, "Phase-conjugated Twin Waves for Communication beyond the Kerr Nonlinearity limit," Nature Photonics, vol. 7, pp. 560-568, May 2013.

[25] V. E. Zakharov and A. B. Shabat, "Exact Theory of Two-dimensional Self-focusing and One-dimensional Self-modulation of Waves in Nonlinear Media," Soviet Physics - JETP, vol. 34, pp. 62-69, Jan. 1972.

[26] M. J. Ablowitz, D. J. Kaup, A. C. Newell, and H. Segur, "The Inverse Scattering Transform-Fourier Analysis for Nonlinear Problems," Stud. Appl. Math., vol. 53, pp. 249-315, 1974.

[27] A. Hasegawa and T. Nyu, "Eigenvalue Communication," J. Lightwave Technol., vol. 11, pp. 395-399, Mar. 1993.

[28] E. G. Turitsyna and S. K. Turitsyn, "Digital signal processing based on inverse scattering transform," Opt. Lett., vol. 38, no. 20, pp. 4186-4188, Oct. 2013.

[29] M. I. Yousefi and F. R. Kschischang, "Information Transmission Using the Nonlinear Fourier Transform, Part I: Mathematical Tools," IEEE Trans. Inf. Theory, vol. 60, no. 7, pp. 4312-4328, Jul. 2014.

[30] M. I. Yousefi and F. R. Kschischang, "Information Transmission Using the Nonlinear Fourier Transform, Part II: Numerical Methods," IEEE Trans. Inf. Theory, vol. 60, no. 7, pp. 4329-4345, Jul. 2014.

[31] M. I. Yousefi and F. R. Kschischang, "Information Transmission Using the Nonlinear Fourier Transform, Part III: Spectrum Modulation," IEEE Trans. Inf. Theory, vol. 60, no. 7, pp. 4346-4369, Jul. 2014.

[32] S. Hari, F. Kschischang, and M. Yousefi, "Multi-eigenvalue Communication via the Nonlinear Fourier Transform," in 27th Biennial Symposium on Communications (QBSC), Kingston, ON, Canada, pp. 92-95, Jun. 2014.

[33] E. Meron, M. Feder, and M. Shtaif, "On the Achievable Communication Rates of Generalized Soliton Transmission Systems," 2012. Available: http://arxiv.org/abs/1207.0297

[34] J. E. Prilepsky, S. A. Derevyanko, and S. K. Turitsyn, "Nonlinear Spectral Management: Linearization of the Lossless Fiber Channel," Opt. Express, vol. 21, no. 20, pp. 24344-24367, Oct. 2013.

[35] J. E. Prilepsky, S. A. Derevyanko, K. J. Blow, I. Gabitov, and S. K. Turitsyn, "Nonlinear Inverse Synthesis and Eigenvalue Division Multiplexing in Optical Fiber Channels," Phys. Rev. Lett., vol. 113, art. no. 013901, Jul. 2014

[36] S. T. Le, J. E. Prilepsky, and S. K. Turitsyn, "Nonlinear Inverse Synthesis for High Spectral Efficiency Transmission in Optical Fibers," Opt. Express, vol. 22, no. 22, pp. 26720-26741, Nov. 2014.

[37] S. T. Le, J. E. Prilepsky, and S. K. Turitsyn, "Nonlinear Inverse Synthesis Technique for Optical Links with Lumped Amplification," Opt. Express, vol. 23, no. 7, pp. 8317-8328, Apr. 2015.

[38] H. Bülow, "Experimental Demonstration of Optical Signal Detection Using Nonlinear Fourier Transform," J. Lightwave Technol., vol. 33, no. 7, pp. 1433-1439, Feb. 2015.

[39] J. D. Ania-Castanon, et al. "Ultralong Raman fibre lasers as virtually lossless optical media," Phys. Rev. Lett., vol. 96, no. 2, art. no. 023902, Jan. 2006.

[40] T. J. Ellingham, et al., "Quasi-Lossless Optical Links for Broad-Band Transmission and Data Processing," IEEE Photon. Technol. Lett. vol. 18, no. 1, pp. 268-270, Jan. 2006.

[41] G. E. Falkovich, V. Lebedev, I. Kolokolov and S. K. Turitsyn, "Statistics of Soliton-bearing Systems with Additive Noise," Phys. Rev. E, vol. 63 , art. no. 025601(R), Jan. 2001.

[42] S. A. Derevyanko, S. K. Turitsyn, and D. A. Yakushev, "Non-Gaussian Statistics of an Optical Soliton in the Presence of Amplified Spontaneous Emission," Opt. Lett., vol. 28, no. 21, pp. 2097-2099, Nov. 2003.

[43] S. A. Derevyanko, S. K. Turitsyn, and D. A. Yakushev, "Fokker-Planck Equation Approach to the Description of Soliton Statistics in Optical Fiber Transmission Systems", J. Opt. Soc. Am. B, vol. 22, no. 4, pp. 743-752, Apr. 2005.

[44] A. Lapidoth, S. M. Moser, "Capacity Bounds via Duality with Applications to Multiple-antenna Systems on Flat-fading Channels", IEEE Trans. Inf. Theory, vol. 49, no. 10, pp. 2426-2467, Oct. 2003.

[45] A. Mecozzi and M. Shtaif, "On the capacity of intensity modulated systems using optical amplifiers", IEEE Photon. Technol. Lett, vol. 13, no. 9, pp. 1029-1031, Sept. 2001. 